\title{
Fondements et implications de la diversité organisationnelle au sein du commerce équitable
}

Benjamin Huybrechts - Centre d'Economie Sociale - Université de Liège ${ }^{1}$

Sart Tilman B33 Boîte 4 - 4000 LIEGE - BELGIQUE

A paraître dans les «Annales d'économie publique, sociale et coopérative », Volume 78, n², CIRIEC International, 2007

\section{Résumé :}

Cet article a pour but d'étudier le paysage organisationnel d'un secteur en pleine expansion : celui du commerce équitable. Plus particulièrement, nous cherchons à comprendre les raisons qui expliquent la diversité des formes organisationnelles des acteurs qui s'investissent dans cette filière. A cette fin, nous commençons par décomposer le concept de commerce équitable en quatre grands types de biens. Ensuite, en lien avec ces quatre types de biens, nous tentons de comprendre les facteurs d'émergence des différents acteurs de la filière. Enfin, nous étudions la répartition des rôles entre ces différents acteurs et évoquons quelques pistes quant à l'évolution de ce secteur. Nous ciblons notre analyse sur les organisations des pays « du Nord », en illustrant notre propos par des exemples d'organisations belges.

\section{Mots-clés :}

Commerce équitable - diversité organisationnelle - économie sociale - responsabilité sociale des entreprises - Belgique

\section{Catégories JEL :}

H44 - L22 - L31

\footnotetext{
${ }^{1}$ b.huybrechts@ulg.ac.be - L'auteur tient à remercier Jacques Defourny et Sybille Mertens pour leurs nombreuses relectures et pour leurs conseils avisés.
} 


\section{Fondements et implications de la diversité organisationnelle au sein du commerce équitable}

Depuis plusieurs décennies, le commerce équitable constitue un phénomène économique et social dont la notoriété et le succès commercial sont croissants, bien que le poids économique de cette filière reste encore marginal (Moore, 2004 ; Poncelet et al, 2005). Peu de recherches, toutefois, se sont penchées de manière approfondie sur les acteurs de ce secteur.

Jusqu'ici, les travaux économiques ont tenté de décrypter ces acteurs sous l'angle des théories relatives aux «non-profit organizations », étant donné la nature associative des pionniers du commerce équitable (Delaval, 2003; Becchetti et Rosati, 2005 ; Poncelet et al., 2005). Ces dernières années, cependant, avec le développement de la labellisation et l'extension de la filière à la grande distribution, la diversité organisationnelle des organisations de commerce équitable s'est accrue pour inclure un nombre croissant d'entreprises à but lucratif, autant dans la distribution que dans l'importation des produits (Davies et Crane, 2003 ; Renard, 2003). Quant aux organisations fondatrices, on peut observer un certain glissement, si pas vers une logique explicite de profit, du moins vers une certaine accentuation de la dynamique commerciale (Gendron, 2004).

Par conséquent, en vue de comprendre les organisations de commerce équitable (OCE) dans leur complexité et leur diversité, il est important de combiner les arguments de divers champs théoriques relatifs aux différents types de structures (associations, fondations, coopératives, entreprises à but lucratif,...). Pour ce faire, nous adoptons une approche de type néoinstitutionnaliste : «se pencher sur la diversité organisationnelle empiriquement observée et expliquer pourquoi, parmi un ensemble d'arrangement institutionnels alternatifs possibles, telle institution particulière s'est imposée et développée »(Platteau, 1987, p. 7). 
Nous ciblons notre analyse aux OCE des pays du Nord ${ }^{2}$, en essayant d'illustrer notre exposé à travers des exemples provenant essentiellement de la filière du commerce équitable en Belgique. Nous commençons par décomposer les différents types de biens correspondant à la notion de commerce équitable. Cette caractérisation nous permet alors de mieux comprendre les facteurs d'émergence des divers acteurs par rapport à ces différentes dimensions. Dans un troisième temps, nous analysons la répartition actuelle des rôles entre les différents acteurs (y compris l'Etat) ainsi que l'évolution possible de cette répartition.

\section{Décomposition du concept de commerce équitable}

Avant de décomposer le concept de commerce équitable, il est important de le définir brièvement. La définition la plus communément acceptée est celle proposée par le réseau «INE $»^{3}$ en octobre 2001 :

Le Commerce équitable est un partenariat commercial, fondé sur le dialogue, la transparence et le respect, dont l'objectif est de parvenir à une plus grande équité dans le commerce mondial. Il contribue au développement durable en offrant de meilleures conditions commerciales et en garantissant les droits des producteurs et des travailleurs marginalisés, tout particulièrement au Sud de la planète. Les organisations du Commerce équitable (soutenues par les consommateurs) s'engagent activement à soutenir les producteurs, à sensibiliser l'opinion et à mener campagne en faveur de changements dans les règles et pratiques du commerce international conventionnel.

Il ressort de cette définition que le commerce équitable est un concept multidimensionnel, portant à la fois sur une pratique commerciale, sur un projet de développement et sur une

\footnotetext{
${ }^{2}$ Dans cet article, nous parlons des pays du «Nord » et du « Sud » en référence à une division géopolitique de la planète entre les pays industrialisés et les pays en voie de développement. Dans la filière du commerce équitable, des consommateurs sensibilisés au Nord soutiennent le développement de producteurs au Sud à travers l'achat de leurs produits à des conditions «équitables ».

${ }^{3}$ FINE regroupe les principales organisations faîtières du commerce équitable : EFTA (European Fair Trade Association), NEWS (Network European Worldshops), FLO INTERNATIONAL (Fair Trade Labelling Organizations) et IFAT (International Fair Trade Association).
} 
ambition de changer les règles du commerce mondial. Si le commerce équitable pourrait être décomposé de différentes manières, en fonction des angles d'approche, nous avons quant à nous identifié, d'un point de vue économique, quatre grands types de biens.

\subsection{La production d'un cadre de régulation}

Le désir d'une régulation alternative des échanges marchands Nord-Sud, dans un sens plus favorable aux producteurs marginalisés des pays du Sud, est à la base du projet du commerce équitable. En outre, ce cadre normatif n’est pas uniquement destiné à régir les échanges au sein de la filière spécifique du commerce équitable : il a également la vocation de questionner le système économique mondial quant aux impacts de son fonctionnement sur les populations du Sud (Roozen et Van der Hoff, 2001 ; Renard 2003 ; Gendron, 2004). Dans cette perspective, la fonction de régulation exercée par les OCE, même appliquée à un segment très limité du commerce, peut être vue comme un bien public.

En effet, la régulation respecte les deux conditions mises en exergue dans la littérature économique pour caractériser un bien public (Milgrom et Roberts, 1992 ; Stiglitz, 2000). Tout d'abord, il est impossible de priver une partie de la population de l'accès à ce bien (condition de non excludabilité). De plus, la «consommation » de ce bien par un individu n'entraîne pas la diminution de la quantité disponible pour d'autres individus (condition de non rivalité). Les législations par les pouvoirs publics constituent bien sûr la meilleure illustration de la régulation comme bien public. Toutefois, au-delà du lobbying, les exemples d'initiatives spontanées et non étatiques de régulation, comme c'est le cas du commerce équitable, se multiplient au sein de la « société civile»(Abdelgawad, 2003) ${ }^{4}$. Le fait que certains acteurs se spécialisent dans cette

\footnotetext{
${ }^{4}$ On peut mentionner ici d'autres initiatives comme la finance solidaire, le tourisme équitable ou les initiatives de certification environnementale.
} 
fonction de régulation et de contrôle du caractère équitable des biens (notamment les organes de certification $^{5}$ ), confirme qu'il s'agit là d'une fonction à part entière.

\subsection{La sensibilisation et l'éducation}

Distincte de la fonction de régulation, l'action de sensibilisation et d'éducation s'adresse d'abord aux consommateurs et aux citoyens du Nord. L'éducation constitue dans ce cas-ci un bien public, car il s'agit d'une production de service non rivale et non exclusive là où, techniquement, l'exclusion serait possible (Stiglitz, 2000). En effet, la promotion d'une consommation responsable en faveur des producteurs du Sud est «gratuite» et bénéficie à tous les individus concernés par le sort de ces producteurs, même s'ils ne financent pas cette promotion (Ben-Ner et Van Hoomissen, 1991).

L'activité éducative n'est pas assumée par l'ensemble des acteurs aux Nord, mais principalement par les «pionniers » qui voient avant tout dans le commerce équitable un outil de transformation du système économique et de la société (Gendron, 2004). Parmi ces pionniers, on retrouve des acteurs souvent qualifiés d' "ONG», comme par exemple Oxfam ou Traidcraft. Ces acteurs cherchent à mobiliser la population afin de constituer une force d'interpellation et de lobbying qui puisse renforcer la régulation en faveur des producteurs du Sud à partir du cadre normatif proposé. Bien que distincts conceptuellement, les fonctions de régulation et d'éducation sont donc liées et complémentaires.

\subsection{La commercialisation du « produit intrinsèque »}

Si, à la suite de Delaval (2003) et de Becchetti et Rosati (2005), on décompose le produit proposé au sein du commerce équitable, on peut distinguer deux types de biens. Premièrement, le

\footnotetext{
${ }^{5}$ La labellisation équitable est surtout développée pour les produits alimentaires (système FLO), mais a également été initiée pour les organisations commercialisant des produits artisanaux (système IFAT).
} 
produit dans sa partie physique, intrinsèque, abstraction faite des conditions de production, constitue un bien marchand privé. Celui-ci est produit par des agriculteurs ou artisans du Sud et destiné à des consommateurs au Nord. Ces derniers sont financeurs et bénéficiaires en tant que consommateurs, comme ils le sont pour les autres biens de consommation traditionnels. Cette caractérisation du produit intrinsèque est confirmée par le fait que les mêmes produits sont souvent écoulés dans d'autres filières, sans reconnaissance d'une «plus-value équitable ».

\subsection{La plus-value équitable}

La «plus-value équitable » correspond dès lors aux conditions de production spécifiques qui font qu'un bien est qualifié d' «équitable ». Il s'agit en quelque sorte de l'application, au sein de partenariats entre des organisations du Nord et du Sud, du cadre normatif élaboré. En effet, le cadre de régulation est transposé dans un ensemble de critères et d'outils opérationnels dont la mise en œuvre est censée générer une «plus-value » aux niveaux social et environnemental. Ces outils incluent en premier lieu le paiement du «juste prix », généralement plus élevé que le prix du marché et stable dans le temps. En outre, d'autres mécanismes caractérisent l'échange équitable, comme le préfinancement des commandes, la prime destinée à des projets de développement locaux ou encore la stabilité de la relation partenariale (Moore, 2004). Nous ne détaillons pas ici les différents mécanismes du commerce équitable, mais nous retenons que toutes ces caractéristiques constituent un ensemble d'outils visant à générer une plus-value sociale et environnementale pour les producteurs du Sud (Becchetti et Rosati, 2005).

L'application effective des critères du commerce équitable par l'importateur constitue un bien de confiance, c'est-à-dire un bien pour lequel les consommateurs sont confrontés à un problème d' «asymétrie d'information » (Weisbrod, 1988). En effet, la qualité de ce bien est difficilement observable, dans la mesure où, étant donné la distance géographique, les consommateurs au Nord - qui sont les financeurs de ce bien - peuvent difficilement vérifier que les importateurs mettent 
effectivement en œuvre les conditions d'échange annoncées en faveur des producteurs du Sud. Par conséquent, les deux parties ne possèdent pas la même information et l'entreprise qui offre ce bien pourrait être tentée de profiter de cette situation pour diminuer la qualité «équitable » à l'insu du consommateur et du financeur, tel que le décrit Akerlof (1970) dans le cas des voitures d'occasion. Dès lors, les entreprises devront parvenir à convaincre les consommateurs qu'elles sont dignes de confiance, comme nous le verrons dans la suite de cet article.

Comme nous pouvons le voir dans le tableau récapitulatif ci-après, il apparaît que le commerce équitable est composé de différentes dimensions de natures privée, publique et de confiance. Ce caractère multidimensionnel doit être pris en compte pour comprendre la présence d'acteurs de formes différentes. En effet, la nature des biens apparaît comme une caractéristique fondamentale pour expliquer les choix de formes organisationnelles (Ben-Ner et Van Hoomissen, 1991 ; Ben-Ner, 2002 ; Anheier, 2005), choix qui reflètent par ailleurs les priorités des acteurs respectifs par rapport à ces différentes dimensions.

$\underline{\text { Tableau } \mathrm{n}^{\circ} 1 \text { : Les différentes dimensions du commerce équitable }}$

\begin{tabular}{|l|l|l|l|l|}
\hline & \multicolumn{1}{|c|}{ Nature } & \multicolumn{1}{|c|}{ Producteurs } & \multicolumn{1}{c|}{ Bénéficiaires } & \multicolumn{1}{c|}{ Financeurs } \\
\hline $\begin{array}{l}\text { Cadre de } \\
\text { régulation }\end{array}$ & Bien public & $\begin{array}{l}\text { Certificateurs } \\
\text { OCE (Nord) }\end{array}$ & Producteurs (Sud) & $\begin{array}{l}\text { Acteurs certifiés } \\
\text { Etat }\end{array}$ \\
\cline { 3 - 5 } $\begin{array}{l}\text { Sensibilisation, } \\
\text { éducation }\end{array}$ & ------- & $\begin{array}{l}\text { Certaines OCE } \\
\text { (Nord) }\end{array}$ & $\begin{array}{l}\text { Consommateurs } \\
\text { Citoyens (Nord) }\end{array}$ & $\begin{array}{l}\text { Consommateurs } \\
\text { Etat (Nord) }\end{array}$ \\
\hline $\begin{array}{l}\text { Produit } \\
\text { intrinsèque }\end{array}$ & Bien privé & $\begin{array}{l}\text { Producteurs } \\
\text { (Sud) }\end{array}$ & $\begin{array}{l}\text { Consommateurs } \\
\text { (Nord) }\end{array}$ & $\begin{array}{l}\text { Consommateurs } \\
\text { (Nord) }\end{array}$ \\
\hline $\begin{array}{l}\text { Plus-value } \\
\text { équitable }\end{array}$ & Bien de confiance & $\begin{array}{l}\text { OCE (Nord) } \\
\text { Producteurs }\end{array}$ & Producteurs (Sud) & $\begin{array}{l}\text { Consommateurs } \\
\text { (Nord) }\end{array}$ \\
\hline
\end{tabular}

\section{Facteurs d'émergence des différents acteurs}

La mise en évidence des différents biens liés au commerce équitable permet de comprendre pourquoi les acteurs de cette filière ont émergé sous différentes formes. En effet, la diversité des 
formes organisationnelles est directement liée à l'hétérogénéité de la demande en termes d'attributs des produits (Handy, 1997 ; Ben-Ner, 2002). Nous passons en revue trois grands types de statuts observables au sein du commerce équitable : les associations, les coopératives et les entreprises à but lucratif. Il s'agit là des catégories-types les plus souvent proposées par la littérature économique (Hansmann, 1996), bien que leurs délimitations soient floues (Anheier et Ben-Ner, 1997) et qu'elles ne reflètent qu'imparfaitement une riche diversité organisationnelle, qui s'étend tout au long d'un « continuum de lucrativité » (Levi, 1998 et 2005).

\subsection{Les associations}

Le commerce équitable a été initié par des associations et des fondations ${ }^{6}$ sans but lucratif, qui, à l'heure actuelle, demeurent encore fort présentes dans la filière ${ }^{7}$. Ces organisations sont caractérisées par le fait qu'il n'y a aucun «propriétaire » qui puisse s'approprier le surplus résiduel -le bénéfice qui reste une fois que toutes les charges ont été acquittées- (Hansmann, 1980 ; Gui, 1991) et que le droit de contrôle résiduel -la prise de décision ultime- échoit à une autre catégorie d'acteurs que les pouvoirs publics (Anheier et Seibel, 1990 ; Mertens, 1999).

Le fait que les pionniers de ce mouvement aient été, pour la plupart, des organisations sans but lucratif, peut s'expliquer de diverses manières. Considérons d'abord les facteurs qui expliquent l'apparition d'une offre de commerce équitable sous une forme non lucrative. Pour commencer, il est probable que les initiateurs des OCE, comme «entrepreneurs idéalistes », aient trouvé dans la forme non lucrative une structure bien adaptée à la poursuite de leur idéal (James, 1998). La forme associative constituerait en effet un moyen efficace de préserver la mission par rapport à

\footnotetext{
${ }^{6}$ Les fondations étant peu nombreuses et absentes du secteur du commerce équitable en Belgique, nous nous limiterons, dans la suite de notre exposé, aux associations sans but lucratif.

${ }^{7}$ En étudiant les structures des différents membres de l'IFAT (fédération internationale regroupant des organisations de producteurs et d'importateurs principalement actifs dans l'artisanat), nous avons identifié, en 2005, 64\% des OCE au Nord ayant le statut d'associations sans but lucratif. La proportion est de $56 \%$ en Europe contre $84 \%$ en Amérique du Nord (sur un échantillon total de 74 organisations).
} 
toute autre considération (Rose-Ackermann, 1987) ou toute tentative d'appropriation du surplus résiduel par une catégorie d'acteurs définie (Hansmann, 1996).

Quant à la demande de commerce équitable sous une forme non lucrative, elle semble tout d'abord liée au fait que la population a des préférences relativement hétérogènes au niveau idéologique. En effet, tandis que certains consommateurs estiment que le commerce mondial est déjà suffisamment régulé, d'autres ne sont pas de cet avis et voudraient des garanties plus élevées en termes de standards sociaux et environnementaux de production. Or l'Etat, d'après certaines théories du «public choice», laissera inévitablement une partie de la population insatisfaite en ce qui concerne son action de régulation (Weisbrod, 1975 ; Kingma, 1997). Il est à noter que l'action publique de régulation s'entend ici à un niveau supranational (Union Européenne, OMC, FMI,...), ce qui accroît encore l'hétérogénéité des préférences de la population et la distance entre ces instances et les attentes des citoyens.

Etant donné l'incapacité apparente des pouvoirs publics nationaux et supranationaux («government failure ») à répondre à cette demande particulière d'un commerce mondial plus équitable, les consommateurs insatisfaits ont dû organiser eux-mêmes ce service. Ces initiatives citoyennes ont pris la forme de structures privées (non publiques) à vocation collective, étant donné les dimensions de bien public que nous avons mises en exergue (régulation et sensibilisation). Cette dimension publique est plus à même d'être assumée par des associations sans but lucratif, qui peuvent plus facilement remédier au problème du «passager clandestin » (Hansmann, 1980) et qui peuvent mobiliser des ressources non marchandes (subsides, dons) et non monétaires (bénévolat) (Laville et Nyssens, 2001). C'est ce qui explique d'ailleurs que les labellisateurs du commerce équitable, en charge du volet de régulation, conservent à présent souvent (du moins en partie) la forme associative pour préserver leur mission sociale (Courville, 2003), malgré une tendance à l'accentuation de leur dimension commerciale (Gendron, 2004). 
En résumé, à la suite de Rose-Ackerman (1987), l'hétérogénéité idéologique semble à la base à la fois de la demande et de l'offre de commerce équitable sous une forme non lucrative.

Le développement du commerce équitable par des structures sans but lucratif semble en outre découler d'une construction conjointe de l'offre et de la demande. C'est ce que Ben-Ner et Van Hoomissen (1991) identifient comme le processus de «demand creating supply» à la base de la création de nombreuses associations. Etant donné l'asymétrie d'informations concernant les conditions de production des biens, les premiers consommateurs désirant des garanties par rapport à des conditions de production «équitable » n'ont eu d'autre choix que de mettre euxmêmes en place l'offre de ce service d'une manière qui minimise les risques d'opportunisme. En contrôlant eux-mêmes la production, ils ont pu maximiser la qualité du service dont ils sont demandeurs (Ben-Ner et Van Hoomissen, 1991 ; Anheier, 2005).

Enfin, au-delà de ces consommateurs initiaux, les fondateurs ont dû convaincre le grand public de leur crédibilité. En effet, en l'absence initiale d'un label ou d'une reconnaissance par l'Etat, la légitimité de la filière a reposé fortement sur la capacité des OCE à conquérir la confiance des consommateurs, dans un contexte d'asymétrie d'information induit par la distance géographique entre les bénéficiaires (producteurs au Sud) et les financeurs (consommateurs au Nord). Ces derniers ne pourraient effectivement vérifier la réalisation par l'OCE de la plus-value sociale annoncée qu'en assumant des coûts de transaction élevés (missions régulières dans les communautés du Sud).

Or, de par l'absence d'objectif de profit et l'interdiction de redistribuer celui-ci, les organisations sans but lucratif apparaissent bien placées pour revendiquer la poursuite «non-opportuniste » d'objectifs difficilement vérifiables (Hansmann, 1980 et 1988 ; Gui, 1991), comme c'est le cas pour la «plus-value équitable». Les associations peuvent ainsi dépasser l'échec du marché («market failure ») décrit par Akerlof (1970) : là où les entreprises auraient intérêt à ne pas 
respecter les critères de qualité annoncés pour accroître leur marge bénéficiaire, les non-profit organizations peuvent faire valoir la contrainte de non distribution du profit comme un signal de confiance. De plus, les associations peuvent utiliser des garanties additionnelles pour renforcer leur capital de confiance, comme par exemple la présence de différentes parties prenantes («stakeholders »), représentant des intérêts divers, ainsi que de travailleurs bénévoles, dans les organes de décision (Enjolras, 2000 ; Ben-Ner et Gui, 2000).

\subsection{Les coopératives}

Alors que la forme coopérative est relativement répandue parmi les organisations de producteurs au Sud, on assiste, depuis quelques années, à une multiplication de coopératives de commerce équitable au Nord ${ }^{8}$ (Develtere et Pollet, 2005). Il peut s'agir d'organisations ayant opté dès le départ pour ce statut (par exemple l'OCE américaine Equal Exchange), ou bien d'organisations qui, à partir d'une forme associative, ont voulu se doter d'une structure plus typiquement commerciale (c'est par exemple le cas des OCE belges Oxfam-Wereldwinkels et Miel Maya).

Quelles sont les raisons qui expliquent l'émergence de ces organisations sous un statut coopératif plutôt qu'associatif ? Tout d'abord, certaines recherches ont montré que le capital de confiance dont jouissent les associations peut également s'appliquer, dans une certaine mesure, aux coopératives (Spear, 2000; Mertens, 2005). Ces dernières seraient capables, à l'instar des organisations sans but lucratif, à dépasser simultanément les échecs du marché (Hansmann, 1996 et 1999) et de l'Etat (Spear, 2005). Dans le cas du commerce équitable, les coopératives seraient ainsi capables à la fois d'assumer les dimensions publiques (sensibilisation et régulation) et de garantir l'absence de comportement opportuniste dans la commercialisation des produits.

\footnotetext{
${ }^{8}$ Selon notre étude citée précédemment, $22 \%$ des OCE du Nord affiliées à l'IFAT ont le statut de coopératives (la proportion est similaire en Europe et en Amérique du Nord).
} 
Ce dernier constat doit toutefois être nuancé par le fait que les coopératives peuvent générer du profit et le redistribuer à leurs membres : le service aux membres constitue, en théorie, leur finalité première. Néanmoins, de plus en plus de coopératives poursuivent, en plus du service à leurs membres, un but explicite de service à la collectivité (Monnier et Thiry, 1997 ; Borzaga et Spear, 2004). Nous émettons l'hypothèse que c'est le cas des coopératives de commerce équitable, dans la mesure où les producteurs du Sud restent normalement les premiers bénéficiaires de l'activité. Quant aux membres, qu'ils soient consommateurs, travailleurs ou financeurs, ne semblent pas revêtir la propriété d'une OCE pour en bénéficier à titre personnel : les produits vendus sont plus chers que dans les circuits traditionnels, les salaires y sont rarement plus élevés qu'ailleurs et le retour financier est généralement très faible (Tadros et Malo, 2002).

Au-delà des avantages qui semblent similaires à ceux des associations sans but lucratif, il s'avère que la forme coopérative permet une plus grande marge de manœuvre commerciale (Hansmann, 1999 ; Levi, 2005). En effet, les coopératives peuvent plus facilement accéder à des capitaux (que ce soit via leurs membres ou auprès des institutions financières), et sont moins susceptibles d'être accusées de concurrence déloyale, entre autres parce que leur activité de production n'est normalement pas subsidiée par les pouvoirs publics (Mertens, 2005). Ainsi, si l'on regarde les OCE coopératives en Belgique, il apparaît que le choix de la structure coopérative (à chaque fois au départ d'une forme associative) a souvent été guidé par la volonté de se mettre à l'abri du point de vue de la fiscalité et des subsides. Cet exemple démontre l'influence de l'environnement économique et légal dans le choix des formes organisationnelles, tel que le postule l'approche néo-institutionnelle (Williamson, 1985), et ce particulièrement en ce qui concerne les coopératives (Platteau, 1987 ; Nilsson, 1999 ; Borzaga et Spear, 2004).

Malgré leurs différences, les coopératives et les associations semblent avoir un grand nombre de traits communs, en premier lieu le fait de ne pas être publiques et de ne pas avoir la recherche de 
profit comme finalité première (Defourny et Develtere, 1999). Nous avons vu, en effet, que les OCE associatives et coopératives semblent toutes deux pouvoir remédier aux incapacités du marché et de l'Etat à répondre à la demande d'un commerce plus équitable. Cette trajectoire commune plaide en faveur d'une approche intégrée du «Third Sector » (Gui, 1991) ou, selon la terminologie latine, de «l'économie sociale », c'est-à-dire l'ensemble des activités économiques exercées par les associations, les coopératives et les mutualités ${ }^{9}$ (Defourny et Develtere, 1999).

A travers le rôle pionnier des associations et des coopératives, certains auteurs considèrent que l'économie sociale constitue en quelque sorte le berceau du commerce équitable, dont elle partage la mission: la poursuite de l'activité économique comme moyen pour réaliser des objectifs sociétaux (Lévesque, 2004). Nous tentons de résumer, dans le tableau suivant, les arguments qui expliquent la création de cette filière par des organisations d'économie sociale.

Tableau $\mathrm{n}^{\circ}$ 2: Récapitulatif des arguments théoriques expliquant la provision initiale de commerce équitable par des associations et des coopératives

\begin{tabular}{|c|c|c|}
\hline $\begin{array}{c}\text { Dimension } \\
\text { Production et contrôle du } \\
\text { cadre normatif }\end{array}$ & Type de bien & Explication théorique \\
\hline Sensibilisation, éducation & Bublic & «Government failure » \\
\hline $\begin{array}{c}\text { Commercialisation du } \\
\text { produit matériel }\end{array}$ & Bien privé & / \\
\hline Plus-value équitable & Bien de confiance & « Market failure » \\
\hline
\end{tabular}

Comme nous pouvons le voir dans la case vide, si la capacité des organisations d'économie sociale à remédier aux échecs de l'Etat et du marché pour produire des biens publics et de confiance est abondamment commentée dans la littérature, il n'en va pas de même pour la production de biens privés classiques. En effet, on considère généralement que ces biens sont nécessairement produits par des entreprises à but lucratif, ou éventuellement par des coopératives

\footnotetext{
${ }^{9}$ Les mutualités, actives dans le domaine de l'assurance-santé, ne sont bien sûr pas présentes au sein du commerce équitable.
} 
(Ben-Ner, 2002). Dans ce cas-ci, néanmoins, il apparaît que le bien privé, en l'occurrence la commercialisation du bien «matériel », n'est pas isolé dans la mesure où il est assorti des autres dimensions identifiées dans le cadre d'une production jointe (Becchetti et Rosati, 2005).

Ce tableau semble également indiquer que les acteurs de l'économie sociale ont été les seuls à pouvoir dépasser simultanément les échecs du marché et de l'Etat dans la production des différentes dimensions du commerce équitable. Or, il est tout à fait possible de produire séparément ces dimensions et de voir l'Etat et les entreprises à but lucratif se cibler sur certaines d'entre elles (respectivement, les dimensions publiques et les activités commerciales). Nous étudions les raisons de la participation des entreprises à but lucratif dans la suite de cette section, avant d'aborder le rôle des pouvoirs publics dans la section suivante.

\subsection{Les entreprises à but lucratif}

La distinction que nous proposons entre la commercialisation des produits et la garantie des conditions de production et d'échange s'est concrétisée à travers le développement de la labellisation, qui a fortement modifié la configuration institutionnelle du secteur. En effet, comme nous l'avons déjà signalé, la fonction de régulation et de contrôle du caractère équitable des échanges a été progressivement isolée et prise en charge par des organismes indépendants. Cette labellisation a permis à toute entreprise active dans la transformation ou la distribution de produits alimentaires de proposer des produits issus du commerce équitable, dans la mesure où c'est le produit qui est certifié et non l'organisation.

En permettant d'insérer la garantie de confiance au sein du produit même, la labellisation a donc permis d'ouvrir la filière à des entreprises commerciales recherchant explicitement le profit. Sans cette labellisation, ces entreprises auraient en effet eu des difficultés à garantir que le surplus de prix payé par le consommateur bénéficierait bien aux producteurs du Sud et non aux actionnaires 
de l'entreprise. C'est ce qui explique pourquoi ces entreprises à but explicitement lucratif étaient auparavant absentes du secteur. L'intérêt manifesté par les entreprises s'est ensuite accru, au fur et à mesure de l'extension de la labellisation à un nombre croissant de produits ${ }^{10}$.

Toutefois, le développement de la labellisation semble être une condition nécessaire mais pas suffisante à la participation d'entreprises à but lucratif : d'autres facteurs doivent être pris en compte pour expliquer leur intérêt dans cette filière. Si les arguments purement commerciaux sont souvent mis en avant pour expliquer l'attrait des entreprises pour cette niche en pleine expansion (Renard, 2003), il paraît également évident que le développement de la notion de responsabilité sociale des entreprises (RSE) a contribué à pousser certaines entreprises à s'investir dans le commerce équitable (Huybrechts, 2006). La participation au commerce équitable comme démarche de responsabilité sociale a d'ailleurs été souhaitée par la plupart des associations et coopératives de la filière (Gendron, 2004). Le commerce équitable constitue donc un exemple de changement des entreprises suite à leur interpellation par des organisations d'économie sociale, dans le cadre de la dénonciation de pratiques commerciales jugées inéquitables (Marsden, 2003 ; Huybrechts et al., 2006).

La RSE n'est cependant pas nécessairement le résultat de pressions extérieures et peut également s'appuyer sur des motivations endogènes (Gond, 2004 ; Pasquero, 2005). On ne peut en effet nier les motivations éthiques personnelles de certains « entrepreneurs idéalistes », sensibilisés au sort des producteurs des pays du Sud. En Belgique par exemple, l'OCE Citizen Dream est un exemple d'entreprise fondée par des entrepreneurs idéalistes (Biélande, 2006). Ces motivations endogènes semblent toutefois plus prégnantes dans les petites structures que dans les grandes entreprises comme les chaînes de grande distribution. Quoi qu'il en soit, la participation des

\footnotetext{
${ }^{10}$ La labellisation concerne actuellement la majorité des produits alimentaires, ainsi que certaines matières premières pour l'artisanat (comme le coton). Si l'on analyse les membres de l'IFAT, qui n'incluent pas les chaînes de grande distribution, il semble que $32 \%$ des organisations engagées dans le commerce équitable aient un statut à but lucratif (bien plus en Europe - 39\% - qu'en Amérique du Nord - 16\% -). Ces chiffres doivent être nuancés par le fait qu'il est parfois très difficile de distinguer lucrativité et non lucrativité, comme l'explique bien Levi (1998).
} 
entreprises à but lucratif au commerce équitable, qu'elle reflète de réelles préoccupations éthiques ou quelle soit guidée par des fins de marketing, semble trouver son origine et s'inscrire pleinement dans la mouvance de la RSE.

En conclusion, on peut affirmer que la filière du commerce équitable évolue vers une diversité organisationnelle croissante. Cette diversité se cristallise autour de deux pôles majeurs : celui de l'économie sociale, qui regroupe les organisations pionnières, et celui des entreprises à but lucratif, dont la présence est appelée à s'étendre dans le contexte de la RSE. Une fois les raisons de cette diversité organisationnelle défrichées, nous pouvons à présent nous interroger sur ses modalités et ses implications au niveau de l'organisation de la filière.

\section{Distribution des rôles et évolution de la filière}

Après avoir étudié les facteurs d'émergence des acteurs du commerce équitable sous différentes formes, il est intéressant de s'interroger sur les conséquences de cette diversité organisationnelle. Autrement dit, quelles sont les implications des différences de structures en termes de participation au commerce équitable ? Nous essayons de souligner les « avantages comparatifs » des différents acteurs (Ben-Ner, 2002), afin de mieux comprendre comment les rôles sont répartis à l'heure actuelle et sont susceptibles d'être répartis à l'avenir au sein de la filière. Cette étude prospective inclut l'apparition d'un quatrième acteur majeur, à savoir les pouvoirs publics.

\section{$\underline{\text { 3.1. Les associations }}$}

En ce qui concerne les activités d'éducation et de sensibilisation (par exemple les campagnes de dénonciation des multinationales), il est probable que cette production continue à être assumée par les associations, étant donné leur enracinement citoyen et leur capacité à s'investir sur des questions idéologiques (James, 1990). Par contre, en ce qui concerne la régulation, les pouvoirs publics semblent de plus en plus sollicités par les acteurs pour prendre position au-delà de la 
simple subsidiation. Ceci est confirmé par les initiatives récentes en vue d'une reconnaissance légale du concept de commerce équitable, notamment en France ${ }^{11}$ et en Belgique ${ }^{12}$.

Pour ce qui est des activités commerciales, les associations disposent d'atouts économiques que d'autres types de structure n'ont pas ou en tout cas moins. Ces atouts incluent l'accès à des ressources spécifiques telles que les dons et le bénévolat. En outre, le capital de confiance auprès des consommateurs les plus sensibilisés demeure important: des études ont montré que ces consommateurs accordent plus de confiance aux associations et à désirent lier leur consommation équitable à un mouvement citoyen (Becchetti et Rosati, 2005). En Belgique par exemple, le réseau des «magasins du monde » mis sur pied par l'association Oxfam continue à voir ses ventes croître grâce à un public sensibilisé, malgré la présence des mêmes produits dans la grande distribution. Cette capacité économique s'inscrit dans une mouvance plus générale du secteur associatif à mobiliser des ressources marchandes (James, 1998 ; Weisbrod, 1998). Dans ce cadre-ci, ce succès commercial semble témoigner d'une volonté d'une partie des consommateurs à ne pas dissocier les différentes dimensions liées au commerce équitable.

Ceci n'empêche pas que les structures sans but lucratif sont également confrontées à de nombreuses limites, tant dans la production de biens publics (Salamon, 1987) que dans l'activité commerciale (Hansmann, 1980). Certains reprochent ainsi aux OCE constituées en associations leur forte subsidiation, leur régime fiscal préférentiel ou encore la faiblesse de l'emploi par rapport au travail bénévole (Hayes, 2005). Ces critiques pourraient amener les pouvoirs publics à restreindre l'activité commerciale des associations, obligeant celles-ci à isoler cette activité de la production de biens publics. C'est cette pression qui, en Belgique, semble à la base de la scission de la plupart des OCE initialement associatives en deux structures (association et coopérative).

\footnotetext{
${ }^{11}$ Après trois années de débats intenses auprès de l'AFNOR (Agence Française de Normalisation), jalonnées par différents rapports (Rapport Herth, loi du 2 août 2005,...), une loi définissant le commerce équitable a été votée en janvier 2006.

${ }^{12}$ En Belgique, la réflexion a été initiée en 2005 et devrait déboucher sur une législation en 2006 : trois propositions de lois émanant de différents partis politiques étaient sur la table au printemps 2006.
} 


\subsection{Les coopératives}

Nous avons vu dans l'étude des facteurs d'émergence que les coopératives semblent bénéficier de certains des atouts des associations en termes de production de biens publics, et ce d'autant plus qu'il y a une tendance générale du mouvement coopératif à endosser des missions de service de l'intérêt général. Cette tendance se concrétise par l'apparition de nouveaux statuts coopératifs dans de nombreux pays (Galera, 2004). Les OCE ayant opté pour la forme coopérative dans ces pays semblent s'inscrire dans ces nouveaux statuts, tel que l'observent Tadros et Malo (2002) dans le cas des OCE québécoises qui ont été reconnues comme « coopératives de solidarité ».

Quant à la gestion de la partie commerciale, la réalité du terrain semble confirmer les atouts commerciaux des coopératives par rapport aux associations, étant donné qu'elles ne sont pas limitées par la contrainte de non distribution des profits (Hansmann, 1996), qu'elles ont un accès plus aisé aux ressources financières (Nilsson, 1999) et que la moindre pression actionnariale peut être un atout pour le développement de l'activité (Levi, 2005). Ainsi, en Belgique, l'OCE Oxfam Fair Trade a considérablement augmenté ses ventes et sa notoriété depuis son lancement comme aile « commerciale » de l'organisation Oxfam-Wereldwinkels (Krier, 2005).

\subsection{Les entreprises à but lucratif}

De manière évidente, les entreprises à but lucratif disposent d'atouts leur permettant d'apporter un dynamisme économique et une visibilité accrus au commerce équitable. C'est d'ailleurs ce qui a motivé les OCE d'économie sociale à leur ouvrir l'accès à la filière : leur rôle dans la distribution des produits a permis d'accroître considérablement les ventes, la notoriété du mouvement et surtout l'impact quantitatif sur les producteurs (Krier, 2005).

En outre, en ce qui concerne leur crédibilité quant à la plus-value équitable, la littérature économique démontre que les associations n'ont pas le monopole de la production de biens de 
confiance (Ortmann et Schlesinger, 1997). Même sans certification externe, les entreprises peuvent construire une réputation en termes de qualité (Milgrom et Roberts, 1992). En Belgique, les initiatives de commerce se voulant éthiques ou équitables se sont multipliées dans le chef d'entreprises qui ne veulent pas s'insérer dans les schémas de certification existants. Cette situation n'est toutefois pas sans créer des tensions avec les acteurs pionniers du mouvement, d'où la demande croissante d'une intervention des pouvoirs publics (Biélande, 2005).

\subsection{Conclusion}

Nous proposons à présent un tableau schématisant, pour chaque type de bien mis en exergue dans la première partie, les atouts et les limites des différents types d'acteurs.

$\underline{\text { Tableau }^{\circ} 3 \text { : Atouts et limites des différents protagonistes }}{ }^{13}$

\begin{tabular}{|c|c|c|c|c|}
\hline Dimension & Associations & Coopératives & $\begin{array}{c}\text { Entreprises à } \\
\text { but lucratif }\end{array}$ & $\begin{array}{c}\text { Pouvoirs } \\
\text { publics }\end{array}$ \\
\hline Cadre normatif & + & \pm & - & + \\
\hline $\begin{array}{c}\text { Sensibilisation, } \\
\text { éducation }\end{array}$ & + & \pm & - & \pm \\
\hline $\begin{array}{c}\text { Commercialisation } \\
\text { du produit matériel }\end{array}$ & \pm & + & + & - \\
\hline $\begin{array}{c}\text { Plus-value } \\
\text { équitable }\end{array}$ & + & + & \pm & \pm \\
\hline
\end{tabular}

Comme nous pouvons le voir, des complémentarités évidentes se dégagent entre les différents acteurs. Une première complémentarité est observable entre les associations et les coopératives, tel que l'atteste le couplage de plus en plus répandu de ces deux structures dans le chef des OCE pionnières. Ensuite, la labellisation des produits alimentaires constitue une illustration d'une dynamique complémentaire entre les entreprises à but lucratif et les organisations d'économie sociale, à travers l'apport de pouvoir économique par les premières, couplée à l'apport de

\footnotetext{
${ }^{13}$ Les signes « + » et « - » renvoient aux atouts théoriques de ces différents types d'institutions et ne constituent en aucun cas des jugements normatifs par rapport à l'activité des organisations concernées.
} 
crédibilité par les secondes. Enfin, d'autres complémentarités peuvent être établies entre les organisations d'économie sociale (en particulier les associations) et les pouvoirs publics (financement de la sensibilisation, reconnaissance du cadre normatif,...) ainsi qu'entre ces derniers et les entreprises à but lucratif (reconnaissance légale du commerce équitable ouverte aux entreprises, préservation d'une concurrence « loyale » dans la filière,...).

La réalité du terrain démontre toutefois également des relations de concurrence, voire de confrontation entre les acteurs (Huybrechts et al., 2006). Ceci s'explique par le fait que, pour certaines dimensions, les acteurs sont également en concurrence (en particulier en ce qui concerne la dimension commerciale, mais également dans la provision des biens publics).

\section{Conclusion générale}

Tout au long de cet article, nous avons essayé de mieux comprendre le paysage organisationnel commerce équitable, en étudiant et en différenciant leurs raisons d'émergence et leurs atouts au sein de la filière. Bien que la complexité et l'évolution rapide de ce paysage le rendent difficile à cerner, il est possible de retirer plusieurs enseignements de notre analyse.

Tout d'abord, la diversité des acteurs semble s'articuler autour de deux pôles majeurs : celui de l'économie sociale et celui du secteur privé à but lucratif. Initié au sein de la première sphère pour de nombreuses raisons, le commerce équitable s'étend à présent également à la seconde. Cette diversité reflète d'ailleurs la tension permanente inhérente au fair trade, entre les objectifs sociaux (et sociétaux) qui le sous-tendent et la dynamique commerciale qui doit permettre la réalisation de ses objectifs (Gendron, 2004).

Nous avons observé des différences dans les atouts et les rôles des différents acteurs participant au commerce équitable. Ces positionnements amènent ces acteurs à investir de différentes 
manières dans les diverses dimensions du commerce équitable, ce qui induit un certain nombre de complémentarités mais également des dynamiques potentiellement conflictuelles.

Dans ce contexte, il sera intéressant de voir à l'avenir dans quelle mesure l'Etat sera susceptible de jouer un rôle d'arbitrage entre les différents acteurs et, à travers eux, entre les visions qu'ils portent. L'évolution rapide du paysage organisationnel du commerce équitable rend donc nécessaire une actualisation régulière de la recherche en ce domaine. Nous espérons déjà avoir pu fournir, à travers cet article, un canevas institutionnel de base en vue d'études empiriques dans les différents pays où s'est implantée la filière du commerce équitable.

\section{Bibliographie}

ABDELGAWAD W., 2003, "Le commerce équitable et la société civile internationale: une chance pour la mondialisation d'un droit de l'économie solidaire", Revue Internationale de Droit Economique, 2003-1, pp. 197-232

AKERLOF G., 1970, “The Market for 'Lemons': Quality Uncertainty and the Market Mechanism”, Quarterly Journal of Economics, n 84 , 487-500

ANHEIER H. et SEIBEL W., 1990, The Third Sector: Comparative Studies of Non-Profit Organizations, Berlin \& New York: De Gruyter

ANHEIER H. et BEN-NER A., 1997, "Changements dans les délimitations et importances relatives des secteurs privé lucratif, non lucratif, coopératif et public" in L. MONNIER et B. THIRY, éds, Mutations structurelles et intérêt général, Ciriec International, Ouvertures économiques - Jalons, Bruxelles: De Boeck Université

ANHEIER H., 2005, Nonprofit Organizations: Theory, Management, Policy, London \& New York: Routledge

BECCHETTI L. et ROSATI F.C., 2005, "The demand for socially responsible products: empirical evidence from a pilot study on fair trade consumers", Ecineq Working Paper, Society for the Study of Economic Inequality, WP 2005-04

BEN-NER A. et VAN HOOMISSEN T., 1991, "Nonprofit Organizations in the Mixed Economy: A Demand and Supply Analysis", Annals of Public and Cooperative Economics, 62:4, $519-550$

BEN-NER A. et GUI B., 2000, "The Theory of Nonprofit Organizations Revisited", in H.K. ANHEIER et A. BEN-NER, eds., The Study of the Nonprofit Enterprise: Theories and Approaches, New York: Kluwer Academic/Plenum Publishers, 3-26 
BEN-NER A., 2002, "The shifting boundaries of the mixed economy and the future of the nonprofit sector", Annals of Public and Cooperative Economics, 73:1, 5-40

BIELANDE P., 2005, "Le Fairtrade sera-t-il victime de son succès ?", Dossier Alter Business News, $\mathrm{n}^{\circ} 100$, Bruxelles

BIELANDE P., 2006, "Citizen Dream : expérience de distribution des produits équitables issus de l'artisanat par une petite entreprise", Dossier spécial Alter Business News, Bruxelles

BORZAGA C. et SPEAR R., 2004, Trends and challenges for Co-operatives and Social Enterprises in developed and transition countries, Trento: Edizione 31

COURVILLE S., 2003, "Social Accountability Audits: Challenging or Defending Democratic Governance?", Law \& Policy, 25:3, 269-297

DAVIES I. et CRANE A., 2003, «Ethical Decision Making in Fair Trade Companies », Journal of Business Ethics, 45, 79-92

DEFOURNY J. et DEVELTERE P., 1999, “Origines et contours de l'économie sociale au Nord et au Sud", in J. DEFOURNY, P. DEVELTERE et B. FONTENEAU, éds., L'économie sociale au Nord et au Sud, Bruxelles: De Boeck Université, 25-56

DELAVAL J.-M., 2003, «Le commerce équitable : un défi lancé à la théorie économique », Working Paper du Centre d'Économie Sociale, Université de Liège

DEVELTERE P. et POLLET I., 2005, "Co-operatives and Fair-Trade", Background Paper, COPAC Open Forum on Fair Trade and Cooperatives, Berlin

ENJOLRAS B., 2000, Nonprofit organizations and economic efficiency, Institute for Social Research, Oslo

GALERA G., 2004, "The evolution of the co-operative form: an international perspective", in Borzaga, C., Spear, R., Trends and challenges for Co-operatives and Social Enterprises in developed and transition countries, Trento: Edizione 31

GENDRON C. 2004, "Un nouveau mouvement socio-économique au cœur d'une autre mondialisation: le commerce équitable", Comparaisons Internationales, $\mathrm{n}^{\circ} 19, \mathrm{CRDC}$, Université du Québec en Outaouais

GUI B., 1991, "The Economic Rationale for the 'Third Sector'. Nonprofit and other Noncapitalist Organizations", Annals of Public and Co-operative Economics, 62:4, 551-572

GOND J.-P., MULLENBACH A., 2004, "Les fondements théoriques de la responsabilité sociétale de l'entreprise", Revue des Sciences de Gestion, n² 205, 93-116

HANDY, F., 1997, "Coexistence of nonprofit, for-profit and public sector institutions", Annals of Public and Cooperative Economics, 68:2, 201-223

HANSMANN H., 1980, “The Role of Non-Profit Enterprise”, Yale Law Journal, 89:5, 835-901 
HANSMANN H., 1988, "The Ownership of the Firm", Journal of Law, Economics and Organizations, $\mathrm{n}^{\circ} 4,267-304$

HANSMANN H., 1996, The Ownership of Enterprise, Cambridge \& London: The Belknap Press of Harvard University Press

HANSMANN H., 1999, "Cooperative Firms in Theory and Practice", The Finnish Journal of Business Economics, n4/1999, 387-403

HAYES M., 2005, "On the efficiency of Fair Trade", Conference Paper, Association for Heterodox Economics, 15-17 July 2005

HUYBRECHTS B., MERTENS S. et XHAUFLAIR V., 2006, "Les interactions entre l'économie sociale et la responsabilité sociale des entreprises. Illustration à travers la filière du commerce équitable", Revue Internationale de Gestion, 31:2, 65-74

HUYBRECHTS B., 2006, "Le commerce équitable comme générateur de responsabilité sociale au sein des échanges commerciaux Nord-Sud", Actes de la 8ième Université de Printemps de l'Audit Social, Dakar, 165-169

JAMES E., 1990, "Economic Theories of the Nonprofit Sector: A Comparative Perspective", in H.K. Anheier and W. Seibel (eds), The Third Sector, Comparative Studies of Nonprofit Organisations, Berlin \& New-York: De Gruyter, 21-30

JAMES E., 1998, "Commercialism among Nonprofits: Objectives, Opportunities and Constraints", in B. WEISBROD, ed, To profit or not to profit - The commercial Transformation of the Nonprofit Sector, New York: Cambridge University Press, 271-285

KINGMA B.R., 1997, "Public good theories of the non-profit sector: Weisbrod revisited", Voluntas, 8:2, 135-148

KRIER J.-M., 2005, "Fair Trade in Europe 2005. Facts and Figures from Fair Trade in 25 European countries, Report for EFTA, FLO, IFAT and NEWS, Brussels

LAVILLE J.-L. et NYSSENS M., 2001, "The Social Enterprise: Toward A Theoretical Approach", in C. BORZAGA et J. DEFOURNY, éds, The Emergence of Social Enterprise, London: Routledge, 312-332

LÉVESQUE B., 2004, "Commerce équitable et économie sociale: une convergence qui s'impose", in L. AUDEBRAND, sous la direction de G. HUOT et L. PROULX, Commerce équitable et économie sociale : le défi de construire un réseau, Actes de Forum, Collection Transfert, Cahiers de l'ARUC, Montréal, 3-19

LEVI Y., 1998, "Coopératives, entreprises sociales et lucrativité", Revue Internationale de l'Economie Sociale (RECMA), n²68, 36-49

LEVI Y., 2005, "How nonprofit and economy can co-exist: a cooperative perspective", ICA XXI International Cooperative Research Conference, Cork 
MARSDEN C., 2003, "Participating in Governance: the Social Responsibility of Companies and NGOs", New Academy Review, 2:1, 4-18

MERTENS S., 1999, "Nonprofit Organisations and Social Economy: Two Ways of Understanding the Third Sector", Annals of Public and Cooperative Economics, 70:3, 501-520

MERTENS S., 2005, "Une explication théorique à l'existence des coopératives agréées et des sociétés à finalité sociale en Belgique", Non-Marchand, Liège

MILGROM P. et ROBERTS J., 1992, Economics, Organization and Management, Englewoods Cliffs, New Jersey: Prentice-Hall International

MONNIER L. et THIRY B., éds., 1997, Mutations structurelles et intérêt général, Ciriec International, Ouvertures économiques - Jalons, Bruxelles: De Boeck Université

MOORE G., 2004, “The Fair Trade movement: parameters, issues and future research”, Journal of Business Ethics, 53(1-2), 73-86

NILSSON J., 1999, "Co-operative Organisational Models as Reflections of the Business Environments", The Finnish Journal of Business Economics, Liiketaloudellinen Aikakauskirja, $\mathrm{n}^{\circ} 4,449-470$

ORTMANN A. et SCHLESINGER M., 1997, "Trust, repute and the role of non-profit enterprise", Voluntas, 8:2, 97-119

PASQUERO J., 2005, "La responsabilité sociale de l'entreprise comme objet des sciences de gestion : un regard historique", in TURCOTTE M.F. et SALMON A. (éds.), Responsabilité sociale et environnementale de l'entreprise, Sainte Foy: Presses de l'Université du Québec

PLATTEAU J.-P., 1987, «La nouvelle économie institutionnelle et la problématique coopérative », Cahiers de la Faculté des Sciences économiques et sociales de Namur, Série recherches, $\mathrm{n}^{\circ} 79$, Université Notre-Dame de la Paix, Namur

PONCELET M., DEFOURNY J. et DE PELSMACKER P., 2005, "Un commerce équitable entre marché et solidarités", Rapport final, PADD II, Politique Scientifique Fédérale, Bruxelles

RENARD M.-C., 2003, "Fair trade : quality, market and conventions", Journal of Rural Studies, $\mathrm{n}^{\circ} 19,87-96$

ROOZEN N. et VAN DER HOFF F., 2001, L'aventure du commerce équitable. Une alternative à la mondialisation, Paris: JC Lattès

ROSE-ACKERMAN S., 1987, "Altruism, Ideological Entrepreneurs and the Non-profit Firm", Voluntas, 8:2, 120-134

SALAMON L., 1987, "Of Market Failure, Voluntary Failure, and Third-Party Government", Journal of Voluntary Action Research, 16 (1), 29-49

SPEAR R., 2000, “The Co-operative Advantage", Annals of Public and Cooperative Economics, 71:4, 507-523 
STIGLITZ J.E., 2000, Economics of the Public Sector, New York: W.W. Norton \& Company

TADROS C. et MALO M.-C., 2002, "Commerce équitable, démocratie et solidarité : Equal Exchange, une coopérative exceptionnelle au Nord", Nouvelles pratiques sociales, 15:1, 76-97

WEISBROD B.A., 1975, "Toward a Theory of the Voluntary Nonprofit Sector in a Three-Sector Economy", in E.S. PHELPS (ed.), Altruism, Morality and Economic Theory, New-York: Russel Sage Foundation, 171-195.

WEISBROD, B.A., 1988, The Nonprofit Economy, Cambridge: Cambridge University Press

WEISBROD B.A., 1998, "Conclusions and Public-policy Issues: Commercialism and the Road ahead", in B.A. WEISBROD, ed, To profit or not to profit - The commercial Transformation of the Nonprofit Sector, New York: Cambridge University Press, 287-305

WILLIAMSON O., 1985, The Economic Institution of Capitalism, New-York: The Free Press 